11 Kim NW, Piatyszek MA, Prowse KR, Harley CB, West MD, Ho PL. Specific association of human telomerase activity with immortal cells and cancer. Science 1994; 266: 2011-2015.

$12 \mathrm{Wu} X \mathrm{X}$, Huang C, He X, Tian YY, Zhou DX, He Y et al. Feedback regulation of telomerase reverse transcriptase: new insight into the evolving field of telomerase in cancer. Cell Signal 2013; 25: 2462-2468.

13 Kamatani Y, Matsuda K, Okada Y, Kubo M, Hosono N, Daigo Y et al. Genome-wide association study of hematological and biochemical traits in a Japanese population. Nat Genet 2010; 42: 210-215

14 Tefferi A, Lasho TL, Patnaik MM, Finke CM, Hussein K, Hogan WJ et al. JAK2 germline genetic variation affects disease susceptibility in primary myelofibrosis regardless of V617F mutational status: nullizygosity for the JAK2 46/1 haplotype is associated with inferior survival. Leukemia 2010; 24: 105-109.

15 Killela PJ, Reitman ZJ, Jiao Y, Bettegowda C, Agrawal N, Diaz Jr LA et al. TERT promoter mutations occur frequently in gliomas and a subset of tumors derived from cells with low rates of self-renewal. Proc Natl Acad Sci USA 2013; 110: 6021-6026.

c) (\$) $\Theta$ This work is licensed under a Creative Commons AttributionBY NC ND NonCommercial-NoDerivs 3.0 Unported License. To view a copy of this license, visit http://creativecommons.org/licenses/by-nc-nd/3.0/

\title{
Low rate of calreticulin mutations in refractory anaemia with ring sideroblasts and marked thrombocytosis
}

\author{
Leukemia (2014) 28, 1374-1376; doi:10.1038/leu.2014.49
}

Refractory anaemia with ring sideroblasts (RARS) and marked thrombocytosis (RARS-T) was proposed in the World Health Organisation 2001 classification of tumours of haematopoietic and lymphoid tissues and retained as a provisional entity in the 2008 version. ${ }^{1}$ RARS-T is characterised by a high rate of JAK2 ${ }^{\mathrm{V} 617 \mathrm{~F}}$ mutations $^{2-4}$ or the presence of mutations in exon 10 of MPL (myeloproliferative leukaemia). ${ }^{5,6}$ The existence of RARS-T as an entity independent from RARS and essential thrombocythemia (ET) was a matter of debate, as certain specialists favoured the hypothesis that RARS-T was a form of ET with $>15 \%$ of ring sideroblasts in the bone marrow, whereas others thought that RARS-T is derived from RARS with secondary thrombocytosis developing through the acquisition of the JAK2 ${ }^{\mathrm{V} 617 \mathrm{~F}}$ mutation.

We recently demonstrated that RARS-T differed from RARS and ET from a clinical, biological and prognostic point of view. ${ }^{4}$ The presence of high rates of splicing factor 3B subunit 1 (SF3B1) mutations in RARS-T and the absence of these mutations in ET strengthened the hypothesis that RARS-T was a distinct entity. ${ }^{7-9}$

However, the myeloproliferative features of RARS-T are not totally explained by $J A K 2^{\mathrm{V} 617 \mathrm{~F}}$ and MPL ${ }^{\mathrm{W} 515 \mathrm{~L}}$ mutations, as these account for only $50 \%$ and $1 \%$ of RARS-T, respectively. The question remains about other causative mutations responsible for the myeloproliferative part of RARS-T. The challenge is the same in myeloproliferative neoplasms (MPN), as $50-60 \%$ of ET and primary myelofibrosis (PMF) do not present with any JAK2 or MPL mutations.

The recent discovery of a high rate of calreticulin (CALR) mutations in JAK2-non-mutated MPNs was a great step towards better understanding of the molecular pattern of these diseases. Mutations in exon 9 of CALR have been reported as mutually exclusive of JAK2 and MPL mutations, and found in $67-71 \%$ of ET and $56-88 \%$ of PMF with wild-type JAK2 or MPL. ${ }^{10,11}$ These mutations are insertions or deletions leading to a frameshift responsible for modifying the $\mathrm{C}$-terminal part of the protein Following these modifications, the $\mathrm{C}$-terminal part of the protein becomes positively charged and the reticulum targeting KDEL sequence is abrogated, which disturbs its cellular localisation.

Up to now, CALR mutations have only been explored in small RARS-T cohorts, with a mutation frequency ranging from 0 to $12.5 \% .{ }^{10,11}$ The purpose of our study was to analyse the frequency of CALR mutations in a large RARS-T cohort in order to determine whether CALR mutations may also be responsible for thrombocytosis in this disease. We therefore analysed a large cohort of 95 RARS-T patients, with 29 RARS as the control.

According to the WHO 2008 classification, patients are diagnosed with RARS-T if they fulfil the following criteria: i) anaemia (haemoglobin level $<125 \mathrm{~g} / \mathrm{l}$ for females and $<135 \mathrm{~g} / \mathrm{l}$ for males) with erythroid dysplasia and $>15 \%$ ring sideroblasts; ii) thrombocytosis with $>450 \times 10^{9}$ platelets/l; iii) $<5 \%$ blast cells in the bone marrow; iv) presence of large atypical megakaryocytes similar to those observed in $B C R-A B L 1$-negative MPN; v) no secondary cause of ring sideroblasts; and vi) no karyotype abnormalities such as del(5q), $t(3 ; 3)(q 21 ; q 26)$

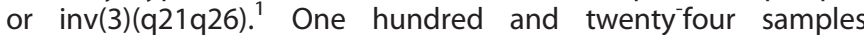
including 95 RARS-T and 29 RARS from seven European centres in three European countries were collected and tested. This cohort has been published previously. ${ }^{4,9}$

For each patient, demographic (gender and age at diagnosis) and biological data (blood cell count, bone marrow exploration, ring sideroblasts, karyotype and molecular explorations) were collected.

The SF3B1 mutations were analysed with a sensitive nextgeneration amplicon deep-sequencing assay (454 Life Sciences, Branford, CT, USA) with a median coverage of 500 reads. This approach was able to detect mutations with a sensitivity below $1 \%{ }^{9}$

The JAK2 $2^{\mathrm{V} 617 \mathrm{~F}}$ mutation was analysed by allele-specific real-time PCR to estimate the allele burden according to the method published by Lippert et al. ${ }^{12}$ with a sensitivity of $1 \%$. JAK2 exon 12 analysis was performed according to the method by Schnittger et al. $^{13}$ and the MPL ${ }^{\text {W515 }}$ mutations were analysed by highresolution melting curve analyses followed by Sanger sequencing if positive, as previously published by Schnittger et al. ${ }^{14}$

The CALR exon 9 mutations were screened by fragment analysis and Sanger sequencing according to the method by Klampfl et al. ${ }^{10}$

Approval for the study was obtained from the ethics committee of each institution and the procedures were carried out in accordance with the Helsinki Declaration of 1975, as revised in 2000.

A total of 124 cases with 95 RARS-T and 29 RARS (including 62 males and 62 females) were recorded in the study, which is, to our best knowledge, the largest series of myelodysplastic syndromes with ring sideroblasts (MDS-RS) studied for CALR mutations. The median age at diagnosis was 74 years and 73 years for the RARS-T and RARS cohort, respectively (Table 1). A karyotype was available in 112 cases (87 RARS-T and 25 RARS). Seventy-five (86.2\%) RARS-T 
Table 1. Demographic and biological characteristics of RARS-T and RARS patients

\begin{tabular}{|c|c|c|}
\hline & RARS-T patients & RARS patients \\
\hline$n$ & 95 & 29 \\
\hline Male (\%) & 45.2 & 65.5 \\
\hline \multicolumn{3}{|l|}{ Age at diagnosis (years) } \\
\hline Median & 74.0 & 73.0 \\
\hline Range & $44.4-88.1$ & 48.4-93.0 \\
\hline $20-50(n)$ & 4 & 2 \\
\hline $50-70(n)$ & 30 & 8 \\
\hline$>70(n)$ & 61 & 19 \\
\hline \multicolumn{3}{|l|}{$W B C\left(\times 10^{9} / I\right)$} \\
\hline Median & 7.6 & 5.5 \\
\hline Range & $2.1-60$ & $1.9-17.3$ \\
\hline \multicolumn{3}{|l|}{$H b(g / l)$} \\
\hline Median & 9.6 & 9.1 \\
\hline Range & $5.1-12.9$ & $6.9-12.8$ \\
\hline \multicolumn{3}{|l|}{ Platelets $\left(\times 10^{9} / \mathrm{l}\right)$} \\
\hline Median & 655 & 274 \\
\hline Range & $455-1500$ & $61-444$ \\
\hline $450-600(n)$ & 45 & \\
\hline$>600(n)$ & 50 & \\
\hline \multicolumn{3}{|l|}{ Ring sideroblasts (\%) } \\
\hline Median & 53.5 & 46.5 \\
\hline Range & $16-97$ & $19-85$ \\
\hline \multicolumn{3}{|l|}{ Karyotype } \\
\hline Tested $(n)$ & 87 & 25 \\
\hline Abnormal $(n)$ & 12 & 6 \\
\hline SF3B1 mutations (\%) & 88.3 & 82.7 \\
\hline Tested $(n)$ & 94 & 29 \\
\hline JAK2 $2^{\mathrm{V} 617 \mathrm{~F}}$ mutations (\%) & 49.4 & 0.0 \\
\hline Tested $(n)$ & 95 & 29 \\
\hline MPL mutations (\%) & 1.1 & 0.0 \\
\hline Tested $(n)$ & 88 & 23 \\
\hline CALR mutations (\%) & 1 & 3.4 \\
\hline Tested $(n)$ & 95 & 29 \\
\hline
\end{tabular}

Abbreviations: CALR, calreticulin; JAK2, Janus kinase 2; MPL, myeloproliferative leukaemia; SF3B1, splicing factor $3 B$ subunit 1; RARS, refractory anaemia with ring sideroblasts; RARS-T, refractory anaemia with ring sideroblasts and marked thrombocytosis; WBC, white blood cells. patients carried a normal karyotype, whereas 12 (13.8\%) patients showed aberrant karyotypes. The rates in RARS-T and RARS patients were comparable.

An SF3B1 mutation was noted in 24 (82.7\%) RARS and 83 (88.3\%) RARS-T confirming that this mutation is highly represented in MDS-RS.

A JAK2 ${ }^{\mathrm{V} 617 \mathrm{~F}}$ mutation was noted in 47 of the 95 RARS-T patients $(49.4 \%)$ but in no RARS patients. The JAK2 ${ }^{\mathrm{V} 617 \mathrm{~F}}$ allele burden was very heterogeneous with a median of $33.3 \%$ (range: $1-92 \%)$. No JAK2 exon 12 mutation (19 tested) was observed, whereas only one $M P L^{\text {W515L }}$ mutation was noted in a patient with RARS-T (88 tested; Table 1 and Figure 1).

A CALR mutation was noted in only one (1\%) RARS-T and one (3.4\%) RARS patient. The CALR mutation found in the RARS-T patient was a 10-bp (1129-1138) deletion located in exon 9. Surprisingly, this patient also presented a JAK2 ${ }^{\mathrm{V} 617 \mathrm{~F}}$ mutation with a low allele burden (4\%) and an SF3B1 mutation. The mutational status of this patient was confirmed by two independent laboratories on two different DNA samples. This patient is a 73-year-old women diagnosed in 2010. At diagnosis, her blood cell count revealed anaemia (haemoglobin $106 \mathrm{~g} / \mathrm{l}$ ) and a marked thrombocytosis $\left(992 \times 10^{9}\right.$ platelets/l). She is currently under cytoreductive and antiplatelet treatment and her last blood count revealed a mild anaemia (haemoglobin $104 \mathrm{~g} / \mathrm{l}$ ) and a platelet count lowered to $600 \times 10^{9} /$.

Among the 29 RARS, we also identified a single case with a $C A L R$ mutation. This unique CALR-positive RARS case was a 70year-old man who had thrombocytopenia and carried an SF3B1 mutation.

In the present study on JAK2 and MPL mutations, our results are in accordance with the usually reported prevalence of $J A K 2^{\mathrm{V} 617 \mathrm{~F}}$ in half of RARS-T and the low frequency of MPL mutations. ${ }^{2-6}$

Recent publications have reported a variable frequency of CALR mutations in MDS-RS: $0 / 6$ to 3/24 in RARS-T, 3/27 in RARS and 0/3 in refractory cytopenia with multilineage dysplasia and ring sideroblasts. ${ }^{10,11}$ However, these results were obtained in small series of patients, thus leading to potential biases. Of note, a high proportion (12.5\%) of CALR mutations in RARS-T in one series of 24 patients contrasts with our results where only one positive case in 95 patients was noted. In particular, one of the three previously reported CALRpositive RARS-T cases did not harbour the SF3B1 mutation, a frequent hallmark of RARS-T. Moreover, in this series, the frequency of the SF3B1 mutation was much lower than in ours (66\% vs $88 \%$ ) suggesting that this CALR-positive SF3B1-negative RARS-T case could be MPN rather than RARS-T, owing to the difficulty of asserting the diagnosis in some cases. Finally, the low rate of CALR mutations in the present RARS-T series is similar (0-3\%) to that reported in chronic myelomonocytic leukaemia, another MDS/MPN. ${ }^{10,11}$

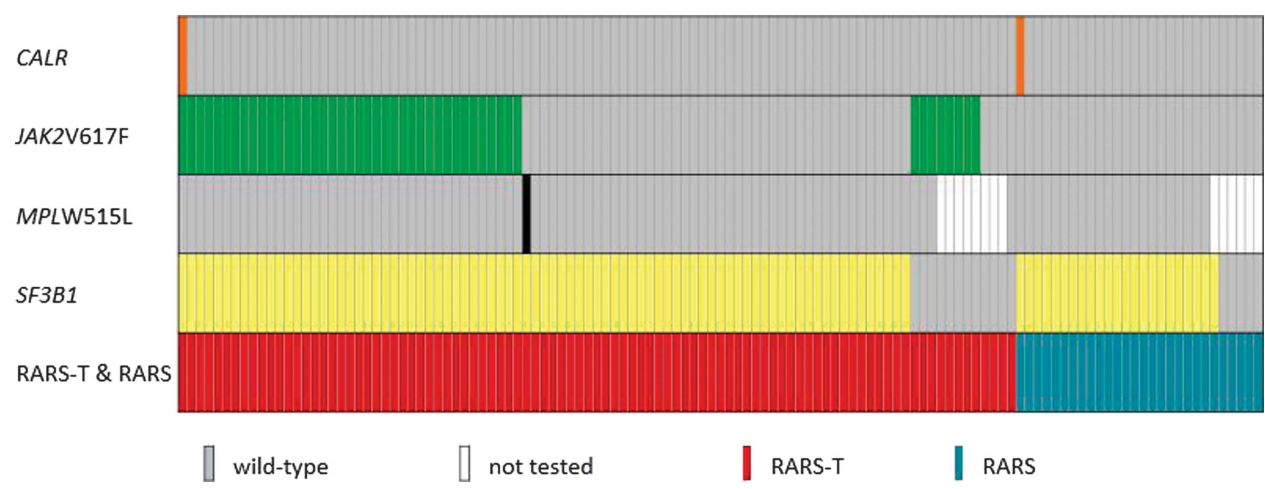

Figure 1. Distribution of mutations in SF3B1, JAK2 ${ }^{\mathrm{V} 617 \mathrm{~F}}, M P L^{\mathrm{W} 515 \mathrm{~L}}$ and CALR mutations in 95 RARS-T and 29 RARS. In RARS-T patients, SF3B1, JAK2 ${ }^{\mathrm{V} 617 \mathrm{~F}}$, MPL and CALR mutations were observed in $88.3 \%, 49.4 \%, 1.1 \%$ and $1 \%$, respectively. In RARS patients, SF3B1, JAK2 ${ }^{\mathrm{V} 617}$, , MPL and CALR $^{\prime}$ mutations were observed in $82.7 \%, 0 \%, 0 \%$ and $3.4 \%$, respectively. 
Among the 29 RARS, we also identified a single case with a CALR mutation, which is consistent with a previous report. ${ }^{11}$

Surprisingly, the CALR-positive RARS-T patient also carried the $J A K 2^{\mathrm{V} 617 \mathrm{~F}}$ mutation, which is to date, the first double-mutant JAK2/ CALR ever described in RARS-T. Indeed, the CALR and JAK2 $2^{\mathrm{V} 617 \mathrm{~F}}$ mutations were described as mutually exclusive in the two recent studies of $>2300$ haematologic cancers. ${ }^{10,11}$ However, another group recently described a case of a JAK2/CALR double mutation in $\mathrm{PMF}^{15}$ which is in keeping with our results and proves that these mutations coexist in rare cases. One can hypothesise that this RARS-T patient with a JAK2/CALR mutation had two different clones. However, owing to absence of frozen progenitor cells, no clonogenic assay was feasible.

The two major types of CALR mutations are type 1 mutations (52 bp deletion; c.1192_1143del) and type 2 mutations (5 bp insertion; c.1154_1155insTTGTC). ${ }^{10}$ The CARL mutation described in our RARS-T is a 10-bp deletion (1129-1138del), which is a rare type of $C A L R$ exon 9 mutation that shifts the reading frame in a similar way to all reported CALR mutations.

Finally, our study showed that CALR mutations are rare in MDSRS and thus do not explain the myeloproliferative part of RARS-T. Approximately $40-50 \%$ of RARS-T are triple-negative for JAK2, MPL and CALR mutations, indicating that further research is required to explain the myeloproliferation in this rare MDS/MPN.

\section{CONFLICT OF INTEREST}

SS and TH declare part ownership of the MLL Munich Leukemia Laboratory. SJ is employed by the MLL Munich Leukemia Laboratory. The remaining authors declare no conflict of interest.

\section{ACKNOWLEDGEMENTS}

We would like to thank the medical doctors from the Haematology Department and Laboratory of the University Hospital of Dijon, the Spanish Group of Hematological Cytology (GECH). EL is grateful to Ms Marina Migeon for expert technical help and to the Tumour Bank of the CHU of Bordeaux. We thank Sylvie Hermouet, medical doctor at the University Hospital of Nantes, for reading the manuscript as well as Philip Bastable for revising the manuscript. This work was supported by grants from the association 'Tulipes contre le cancer' (Châlon s/Saône, Burgundy, France) and from FEHH (Spain) and 2009 SGR 541 (Generalitat de Catalunya).

J Broséus ${ }^{1,2,12,13,14}$, E Lippert $3,4,12,13,14$, AS Harutyunyan ${ }^{5}$ S Jeromin ${ }^{6}$, E Zipperer ${ }^{7}$, L Florensa, ${ }^{8,15}$, JD Milosevic ${ }^{5}$, T Haferlach $^{6}$, U Germing ${ }^{7}$, E Luño ${ }^{9,15}$, S Schnittger ${ }^{6,13}$ R Kralovics ${ }^{5,13}$ and F' Girodon ${ }^{10,11,13,14}$

${ }^{1}$ Service d'Hématologie Biologique, University Hospital, Nancy, France;

${ }^{2}$ Inserm U954, Faculty of medicine, Nancy, France;

${ }^{3}$ Haematology laboratory, University Hospital, Bordeaux, France; ${ }^{4}$ Inserm U1035-Université Bordeaux Segalen, Bordeaux, France;

${ }^{5}$ CeMM Research Center for Molecular Medicine of the Austria Academy of Sciences, Vienna, Austria

${ }^{6}$ MLL Munich Leukemia Laboratory, Munich, Germany;

${ }^{7}$ Department of Haematology, Oncology and Clinical Immunology, Heinrich-Heine-Universität, Düsseldorf, Germany;

${ }^{8}$ Laboratorio Citologia Hematològica, Servicio Patologia, Hospital del Mar, Barcelona, Spain

${ }^{9}$ Servicio de Hematología, Servicio de Salud del Principado de Asturias, Oviedo, Spain;
${ }^{10}$ Haematology Laboratory, University Hospital, Dijon, France and

${ }^{11}$ Inserm U866, Faculty of Medicine Dijon, Dijon, France E-mail: francois.girodon@chu-dijon.fr

${ }^{12}$ These authors contributed equally to this work.

${ }^{13}$ On behalf of MPN\&MPNr-EuroNet (COST Action BM0902).

${ }^{14}$ On behalf of the French Intergroup of MPNs (FIM).

${ }^{15}$ On behalf of the Spanish Group of Hematological Cytology (GECH).

\section{REFERENCES}

1 Vardiman JW, Bennet JM, Bain BJ, Baumann I, Thiele J, Orazi A. Myelodysplastic/ myeloproliferative neoplasm, unclassifiable. In:). Swerdlow $\mathrm{SH}$, Campo $\mathrm{E}$, Lee Harris N, Jaffe ES, Pileri SA, Stein H) (eds). WHO Classification of Tumours of Haematopoietic and Lymphoid Tissues. 4th edn. IARC: Lyon, France, 2008, pp 85-86.

2 Renneville A, Quesnel B, Charpentier A, Terriou L, Crinquette A, Laï JL et al. High occurrence of JAK2 V617 mutation in refractory anemia with ringed sideroblasts associated with marked thrombocytosis. Leukemia 2006; 20 2067-2070.

3 Steensma DP, Caudill JS, Pardanani A, McClure RF, Lasho TL, Tefferi A. MPL W515 and JAK2 V617 mutation analysis in patients with refractory anemia with ringed sideroblasts and an elevated platelet count. Haematologica 2006; 91(12 Suppl): ECR57.

4 Broseus J, Florensa L, Zipperer E, Schnittger S, Malcovati L, Richebourg S et al. Clinical features and course of refractory anemia with ring sideroblasts associated with marked thrombocytosis. Haematologica 2012; 97: 1036-1041.

5 Pardanani AD, Levine RL, Lasho T, Pikman Y, Mesa RA, Wadleigh M et al. MPL515 mutations in myeloproliferative and other myeloid disorders: a study of 1182 patients. Blood 2006; 108: 3472-3476.

6 Schnittger S, Bacher U, Haferlach C, Dengler R, Kröber A, Kern W et al. Detection of an MPLW515 mutation in a case with features of both essential thrombocythemia and refractory anemia with ringed sideroblasts and thrombocytosis. Leukemia 2008; 22: 453-455

7 Visconte V, Makishima H, Jankowska A, Szpurka H, Traina F, Jerez A et al. SF3B1 a splicing factor is frequently mutated in refractory anemia with ring sideroblasts. Leukemia 2012; 26: 542-545.

8 Papaemmanuil E, Cazzola M, Boultwood J, Malcovati L, Vyas P, Bowen D et al. Somatic SF3B1 mutation in myelodysplasia with ring sideroblasts. $N$ Engl J Med 2011; 365: 1384-1395.

9 Broseus J, Alpermann T, Wulfert M, Florensa Brichs L, Jeromin S, Lippert E et al. Age, JAK2(V617F) and SF3B1 mutations are the main predicting factors for survival in refractory anaemia with ring sideroblasts and marked thrombocytosis. Leukemia 2013; 27: 1826-1831.

10 Klampfl T, Gisslinger H, Harutyunyan AS, Nivarthi H, Rumi E, Milosevic JD et al. Somatic mutations of calreticulin in myeloproliferative neoplasms. $N$ Engl J Med 2013; 369: 2379-2390.

11 Nangalia J, Massie CE, Baxter EJ, Nice FL, Gundem G, Wedge DC et al. Somatic CALR mutations in myeloproliferative neoplasms with nonmutated JAK2. N Engl J Med 2013; 369: 2391-2405.

12 Lippert E, Boissinot M, Kralovics R, Girodon F, Dobo I, Praloran V et al. The JAK2V617F mutation is frequently present at diagnosis in patients with essential thrombocythemia and polycythemia vera. Blood 2006; 108: 1865-1867.

13 Schnittger S, Bacher U, Haferlach C, Geer T, Müller P, Mittermüller J et al. Detection of JAK2 exon 12 mutations in 15 patients with JAK2V617F negative polycythemia vera. Haematologica 2009; 94: 414-418.

14 Schnittger S, Bacher U, Haferlach C, Beelen D, Bojko P, Bürkle D et al. Characterization of 35 new cases with four different MPLW515 mutations and essential thrombocytosis or primary myelofibrosis. Haematologica 2009; 94 141-144.

15 Tefferi A, Lasho TL, Finke CM, Knudson RA, Ketterling R, Hanson CH et al. CALR vs JAK2 vs MPL mutated or triple-negative myelofibrosis: clinical, cytogenetic and molecular comparisons. Leukemia 2014; e-pub ahead of print 21 January 2014 doi:10.1038/leu.2014.3. 